\title{
Weak light emission of soft tissues induced by heating
}

\author{
Antonello E. Spinelli \\ Giovanni Durando \\ Federico Boschi
}




\title{
Weak light emission of soft tissues induced by heating
}

\author{
Antonello E. Spinelli, ${ }^{a, \star}$ Giovanni Durando, ${ }^{b}$ and Federico Boschic \\ asan Raffaele Scientific Institute, Experimental Imaging Centre, Milan, Italy \\ 'Istituto Nazionale di Ricerca Metrologica, Ultrasound Laboratory, Torino, Italy \\ 'University of Verona, Department of Computer Science, Verona, Italy
}

\begin{abstract}
The main goal of this work is to show that soft tissue interaction with high-intensity focused ultrasound (HIFU) or direct heating leads to a weak light emission detectable using a small animal optical imaging system. Our results show that the luminescence signal is detectable after $30 \mathrm{~min}$ of heating, resembling the time scale of delayed luminescence. The imaging of a soft tissue after heating it using an HIFU field shows that the luminescence pattern closely matches the shape of the cone typical of the HIFU beam. We conclude that heating a soft tissue using two different sources leads to the emission of a weak luminescence signal from the heated region with a decay half-life of a few minutes (4 to $6 \mathrm{~min}$ ). The origin of such light emission needs to be further investigated. ( 2018 Society of Photo-Optical Instrumentation Engineers (SPIE) [DOI: 10.1117/1.JBO.23.4.046003]
\end{abstract}

Keywords: optical imaging; weak light emission; heat-induced luminescence; high focused ultrasound.

Paper 180004R received Jan. 4, 2018; accepted for publication Mar. 19, 2018; published online Apr. $12,2018$.

\section{Introduction}

Optical imaging is a widely used preclinical and clinical tool to investigate different in vivo biological processes. ${ }^{1}$ In particular, bioluminescence imaging, Cerenkov luminescence imaging (CLI), and radioluminescence imaging (RLI) can be used to detect in vivo weak light emission using a small animal optical imaging system. All these imaging approaches do not require the use of any external light source; more precisely, bioluminescence light is produced during the enzymatic reaction of luciferase with its substrate, typically firefly and D-luciferin.

$\mathrm{CLI}^{2}$ is based on detection of Cerenkov radiation induced by charged particle (typically beta plus or beta minus particles) as they travel into biological tissue with a velocity greater than the speed of light in the tissue. ., $^{3}$

RLI allows the imaging with optical methods of a large variety of radioisotopes, including not just beta but also alpha ${ }^{5}$ and gamma emitters. $^{6-8}$

In this work, we will investigate another source of light emission in soft tissues caused by heating since in preliminary experiments we measured a weak light emission after treating a slab of chicken breast with high-intensity focused ultrasound (HIFU).

HIFU is a noninvasive method developed to treat tumors using an ablative approach, and the basic idea is to use HIFU field to increase the temperature in the focal region to ablate the tumor without damaging the surrounding healthy tissues. ${ }^{9}$ In this paper, we will provide a set of experimental data showing that the interaction with HIFU or direct heating could lead to a weak light emission detectable using a small animal optical imaging system. To the best of our knowledge, there are no studies in the literature regarding the use of HIFU, as pure heating source at high temperature $\left(90^{\circ} \mathrm{C}\right)$, connected with luminescence phenomena.

\footnotetext{
${ }^{*}$ Address all correspondence to: Antonello E. Spinelli, E-mail, spinelli.
} antonello@ hsr.it
As will be described in this paper, the emission of visible light could be interpreted as a mechanism to dissipate energy deposited in the heated tissues. To underline the specific source of light emission, we called this phenomenon the heat-induced luminescence (HIL).

\section{Material and Methods}

\subsection{High Focused Ultrasound System}

The ultrasound field was generated by an HIFU transducer (Type SU-101; Sonic Concepts, Bothell, Washington) in continuous wave mode at $f=1.1 \mathrm{MHz}$ connected to a power amplifier (Type AR 100A250A; Amplifier Research, Souderton, Pennsylvania) and a function generator (Type 33250; Agilent, Santa Clara, California). A mechanical adaptor has been used to connect the $2.5-\mathrm{cm}$-diameter polystyrene tube containing the sample. The HIFU transducer was placed on a $1-\mathrm{cm}$ slab of chicken breast for $1 \mathrm{~min}$. The ultrasound power was $60 \mathrm{~W}$ (intensity $8 \times 10^{3} \mathrm{~W} / \mathrm{cm}^{2}$ ) and spot size was (typically) 1 -mm wide $\times 3$-mmlong. The length of the treatment was chosen to increase the tissue temperature to $90^{\circ} \mathrm{C}$, and these settings were found empirically to obtain a detectable emitting thermal lesion [Fig. 1(b)]. The tissue temperature was measured with an FLIR i7 camera (FLIR Systems, Inc., Wilsonville, Oregon), $140 \times 140$ pixels, field of view (FoV) $25 \mathrm{deg} \times 25 \mathrm{deg}$, accuracy $2 \%$, thermal sensitivity $0.10^{\circ} \mathrm{C}$, and minimum focus distance $=0.6 \mathrm{~m}$.

The slab was then cut along the direction of the HIFU field to image the light emission induced by the HIFU from the slice surface to the focus region.

\subsection{Direct Heating Source}

Direct heating was obtained using a conventional welding device equipped with a thermometer, and the temperature of

\footnotetext{
$1083-3668 / 2018 / \$ 25.00$ @ 2018 SPIE
} 

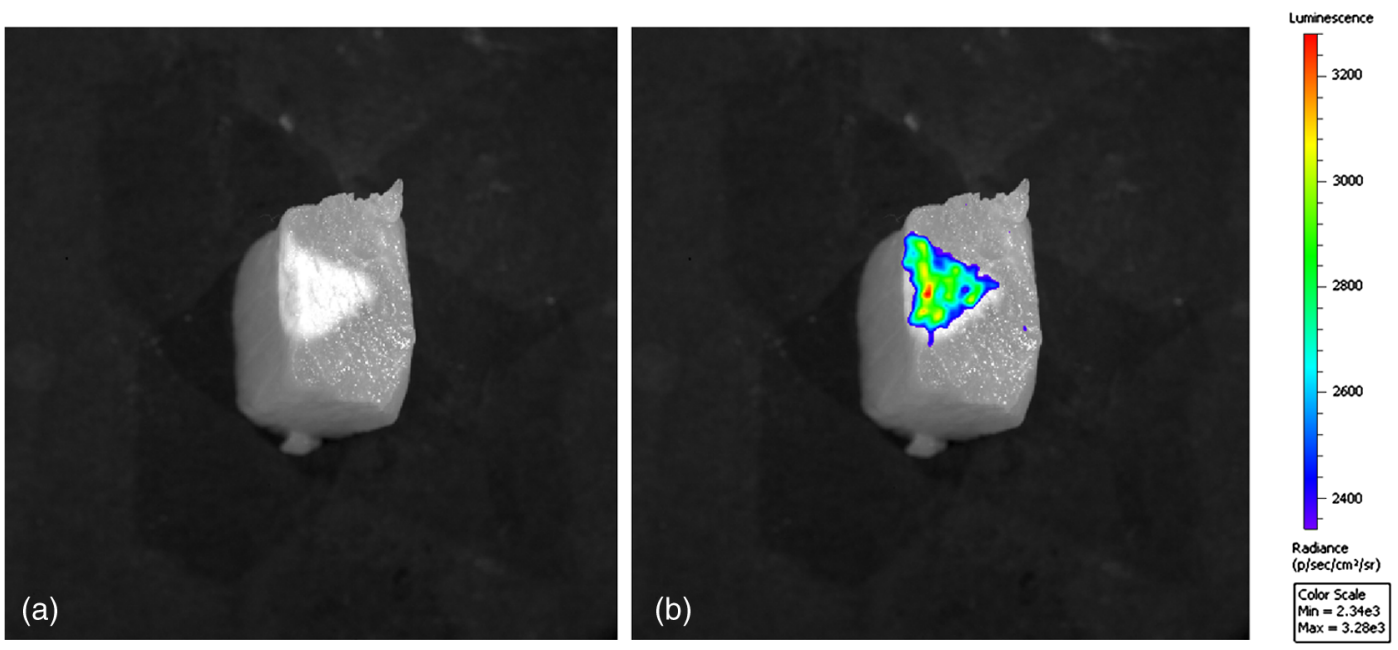

Fig. 1 The image shows the tissue HIL by the HIFU field. As can be seen, there is a good match between the white cone region heated by (a) the HIFU field and (b) the HIL of the sample.

the tip was set to $110^{\circ} \mathrm{C}, 140^{\circ} \mathrm{C}, 180^{\circ} \mathrm{C}$, and $250^{\circ} \mathrm{C}$. The tip was placed with a little pressure on top of a $1-\mathrm{cm}$ slab of chicken breast for 1,10 , and $30 \mathrm{~s}$.

Since different pressure and inclination of the welding probe on the sample may limit the reproducibility of the HIL magnitude, measurements were repeated three times on different regions of the tissue.

\subsection{Heat-Induced Luminescence Images Acquisition}

Optical images of heated tissues were acquired using the IVIS Spectrum optical imager (PerkinElmer, Waltham, Massachusetts). The IVIS Spectrum is based on a cooled $\left(-90^{\circ} \mathrm{C}\right)$, back-thinned, and back-illuminated CCD camera. The CCD has an active array of $1920 \times 1920$ pixels with a dimension of $13 \mu \mathrm{m}$. Images corrected for dark measurements were acquired with Living Image 4.5 (PerkinElmer).

The acquisition parameters for direct heating were: exposure time $=300 \mathrm{~s}$, aperture $f=1$, binning $B=16$, and $\mathrm{FoV}=$ $13.0 \mathrm{~cm}$. Six images were acquired for a total scanning time equal to $30 \mathrm{~min}$.

HIL images when using HIFU heating were acquired using the following setting: exposure time $=120 \mathrm{~s}$, aperture $f=1$, binning $B=16$, and $\mathrm{FoV}=13.0 \mathrm{~cm}$. Fifteen images were acquired for a total scanning time equal to $30 \mathrm{~min}$. A tradeoff was necessary between exposure time and sampling of the HIL; in the case of the HIFU, we opted for a better time sampling.

\subsection{Heat-Induced Luminescence Image Analysis}

HIL image analysis was performed by placing region of interests (ROIs) over the slab of chicken breast, and the total flux $F(t)$ (photons/s) within the ROI was measured. Images were analyzed using Living Image 4.5 (PerkinElmer). The values of the flux measured at different time points were fitted using the following equation:

$F(t)=\left(F_{0}-B\right) e^{-k t}+B$

where the fitting parameters $F_{0}, B$, and $k(1 / \mathrm{min})$ are the intercept, the plateau, and the decay rate constant of the luminescence signal, respectively. The fitting was performed using a nonlinear least square algorithm implemented in GraphPad Prism, version 5.0.

\section{Results}

\subsection{High-Intensity Focused Ultrasound Heating Source}

The image in Fig. 1 shows the tissue HIL caused by the HIFU field in a slab of chicken breast. As can be seen, there is a good match between the cone heated by the HIFU field and the HIL of the sample.

The plot in Fig. 2 shows the decay of the HIL signal, and the half-life resulting from the fit of Eq. (1) was equal to $3.6 \mathrm{~min}$ and the $R^{2}$ value was equal to 0.99 .

\subsection{Direct Heating Source}

The image in Fig. 3 shows the tissue HIL after 30 min when heating at a temperature of $140^{\circ} \mathrm{C}$ for 1,10 , and $30 \mathrm{~s}$ of a small region (3- to 5-mm diameter) of the sample. As can

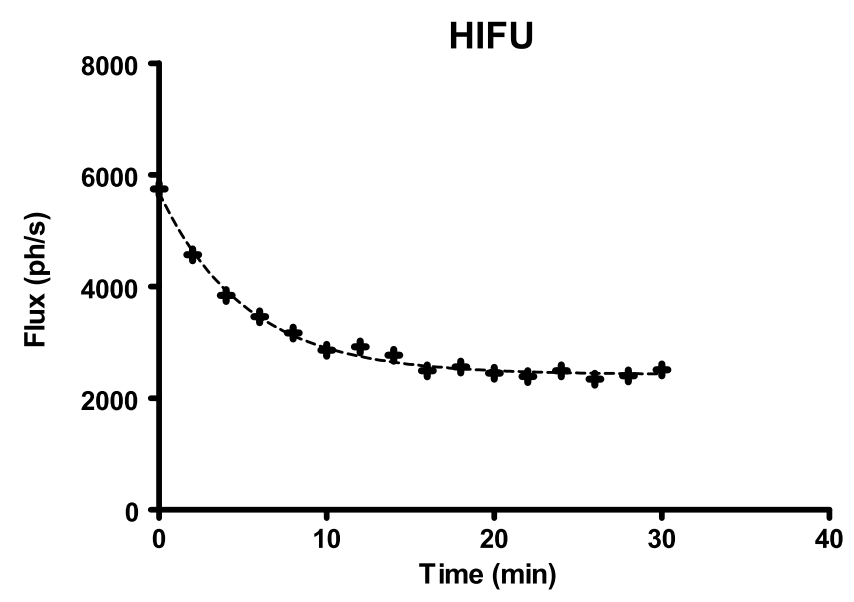

Fig. 2 The plot shows the decay of the HIL signal induced by the HIFU field, and the half-life resulting from the fit was equal to $3.6 \mathrm{~min}$ and the $R^{2}$ value was equal to 0.99 . 


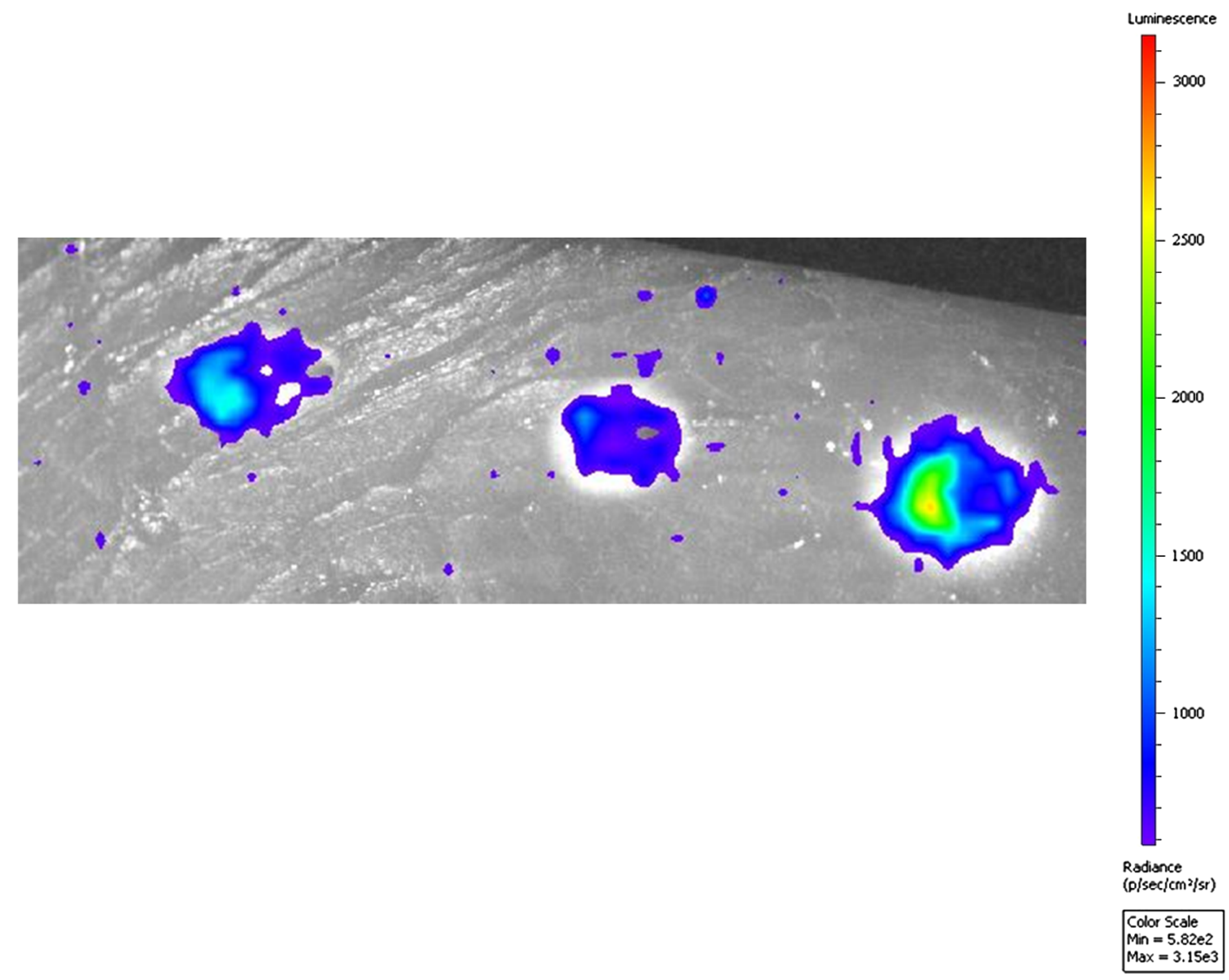

Fig. 3 The image shows an example of tissue HIL obtained after $30 \mathrm{~min}$ from direct heating using a conventional welding device at a temperature of $140^{\circ} \mathrm{C}$ for 1,10 , and $30 \mathrm{~s}$ (from left to right).

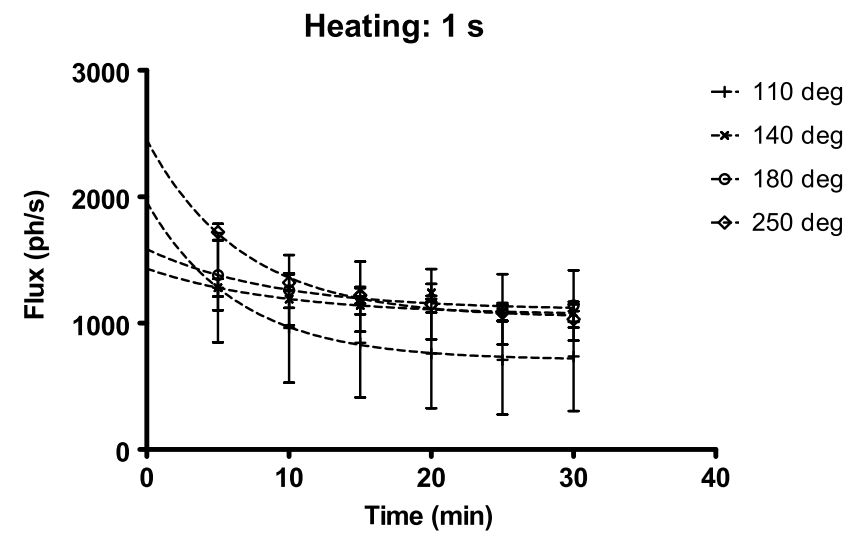

Fig. 4 The plot shows the decay of the HIL signal and the corresponding fitting (dotted line) for the different temperatures and a heating time equal to $1 \mathrm{~s}$. The heating was obtained with a welding device.

be seen, HIL is clearly detectable in the tissue in particular when heating for $30 \mathrm{~s}$.

Considering the small dimension of the heated region and the delay of $30 \mathrm{~min}$ between the heating and image acquisition, it is reasonable to consider the whole sample temperature being equal to the environment (about $25^{\circ} \mathrm{C}$ ). This shows that the HIL signal is, thus, dependent only on the heating time and not on the actual temperature of the spot.

The plots in Figs. 4-6 show the decay of the HIL signal and the corresponding fitting for the different temperatures $110^{\circ} \mathrm{C}$,

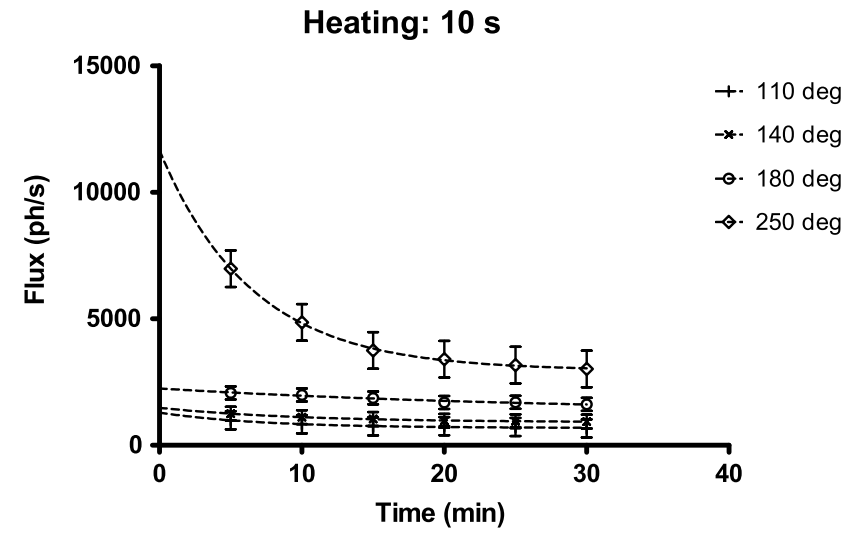

Fig. 5 The plot shows the decay of the HIL signal and the corresponding fitting (dotted line) for the different temperatures and a heating time equal to $10 \mathrm{~s}$. The heating was obtained with a welding device.

$140^{\circ} \mathrm{C}, 180^{\circ} \mathrm{C}$, and $250^{\circ} \mathrm{C}$, and heating times equal to: 1,10 , and $30 \mathrm{~s}$. The fitting $R^{2}$ value is always $>0.95$ showing a good agreement with Eq. (1).

The values of the half-life obtained from the fit can be found in Fig. 7. Except for one outlier, the range of the half-life is between 4 and 6 min. As shown in Fig. 7, the value of the half-life is mainly dependent on the temperature and not on the heating time. More precisely, the half-life is similar for the similar temperature even if the heating times are different. 


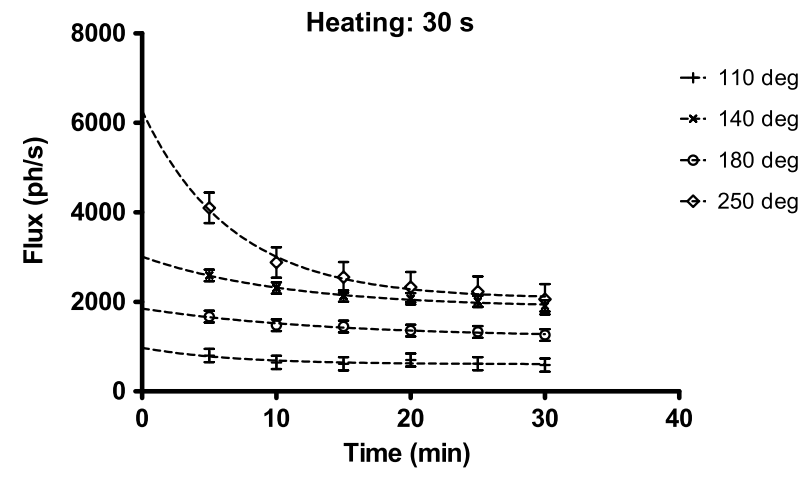

Fig. 6 The plot shows the decay of the HIL signal and the corresponding fitting (dotted line) for the different temperatures and a heating time equal to $30 \mathrm{~s}$. The heating was obtained with a welding device.

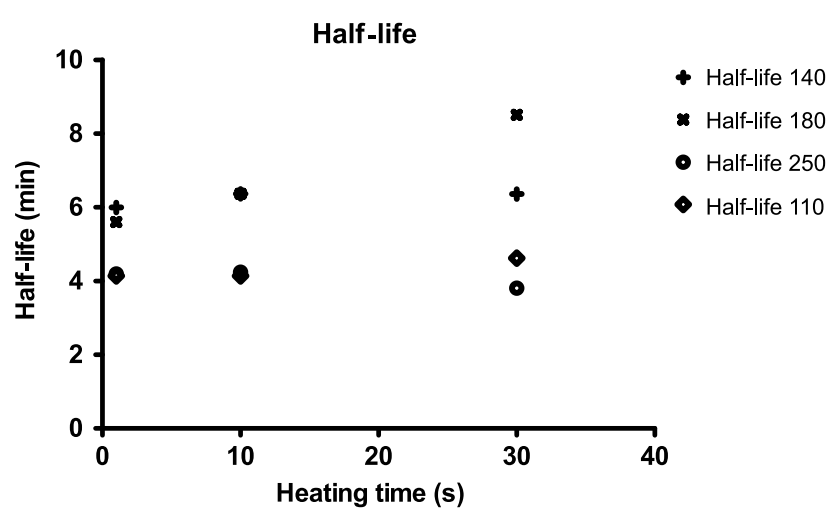

Fig. 7 The plot shows the half-life obtained from fitting the HIL signal decay using Eq. (1). Except for two outliers, the range of the half-life is between 4 and $7 \mathrm{~min}$. The heating was obtained with a welding device.

These data are in agreement with the results obtained using HIFU.

\section{Discussion and Conclusion}

In this work, we performed optical imaging of the weak light emission of a soft tissue induced in tissue by heat; in our knowledge, a detailed analysis of this phenomenon is not reported in literature. The HIL effect might lead to image artifacts when acquiring CLI or RLI images of specimens from surgery ${ }^{10,11}$ removed using a diathermy device or a laser scalpel.

As shown in Fig. 3, the HIL signal is clearly detectable with respect to background even after $30 \mathrm{~min}$ of heating the tissue, and thus, this effect resembles the typical time scale of delay luminescence. We imaged for longer time (30 min equal to 5 half-life) to properly estimate the half-life of the HIL signal with respect to the CLI. More precisely, it is well-known that the half-life of the CLI is equal to the physical half-life of the isotope (e.g., $109 \mathrm{~min}$ for ${ }^{18} \mathrm{~F}$ or $64.2 \mathrm{~h}$ for ${ }^{90} \mathrm{Y}$ ), and thus, a possible approach to limit the contribution of HLI will be to acquire CLI image at later time, e.g., more than $30 \mathrm{~min}$, where the HIL is about $3 \%$ of the initial signal. As mentioned in Sec. 3, the interesting aspect is that the decay pattern of the signal is mainly dependent by the initial temperature while is less dependent on the heating time. We can, thus, claim that the effect is mainly dependent on the initial energy deposition only. However, it is necessary to point out that a longer heating time causes tissue carbonization and this might slightly affect the results by reducing the amount of light emitted. We measured the light emission of carbonized regions (using a small ROI) and we found that the flux was about five times lower than in noncarbonized areas.

The imaging of a soft tissue after heating using an HIFU field shows that the luminescence pattern closely matches the shape of the cone typical of an HIFU beam. The decay of the HIL signal is very similar to the decay obtained with the direct heating of the sample using a conventional welding device.

The detection of the HIL will be heavily dependent on the source intensity (e.g., temperature), tissue thickness, optical properties, and more importantly on the system minimum detectable radiance.

We conclude that heating a soft tissue using two different sources lead to the emission of a weak luminescence from the heated region with a decay half-life of a few minutes (4 to $6 \mathrm{~min}$ ). The origin of such light emission needs to be further investigated; however, a possible explanation of the HIL is the light emission due to the generation of reactive oxygen species after an heat stress. ${ }^{12}$

\section{Disclosures}

The authors have no relevant financial interest to disclose.

\section{References}

1. G. D. Luker and K. E. Luker, "Optical imaging: current applications and future directions," J. Nucl. Med. 49, 1-4 (2008).

2. A. Spinelli et al., "Cerenkov radiation allows in vivo optical imaging of positron emitting radiotracers," Phys. Med. Biol. 55(2), 483-495 (2010).

3. A. Spinelli et al., "Optical imaging of radioisotopes: a novel multimodal approach to molecular imaging," Q. J. Nucl. Med. Mol. Imaging 56(3), 280-290 (2012).

4. A. E. Spinelli and F. Boschi, "Novel biomedical applications of Cerenkov radiation and radioluminescence imaging," Phys. Med. 31, 120-129 (2015).

5. F. Boschi et al., "Optical imaging of alpha emitters: simulations, phantom, and in vivo results," J. Biomed. Opt. 16(12), 126011 (2011).

6. F. Boschi et al., "Combined optical and single photon emission imaging: preliminary results," Phys. Med. Biol. 54(23), L57-L62 (2009).

7. A. Spinelli et al., "Optical imaging of Tc-99m-based tracers: in vitro and in vivo results," J. Biomed. Opt. 16(11), 116023 (2011).

8. F. Boschi et al., "Small-animal radionuclide luminescence imaging of thyroid and salivary glands with Tc99m-pertechnetate," J. Biomed. Opt. 18(7), 076005 (2013)

9. M. C. L. Peek and M. Douek, "Ablative techniques for the treatment of benign and malignant breast tumours," J. Ther. Ultrasound 5, 18 (2017).

10. A. E. Spinelli et al., "Cerenkov and radioluminescence imaging of brain tumor specimens during neurosurgery," J. Biomed. Opt. 21, 050502 (2016)

11. M. R. Grootendorst et al., "Intraoperative assessment of tumor resection margins in breast-conserving surgery using ${ }^{18} \mathrm{~F}$-FDG Cerenkov luminescence imaging: a first-in-human feasibility study," J. Nucl. Med. 58, 891-898 (2017).

12. K. Kobayashi et al., "Biophoton emission induced by heat shock," PLoS One 9(8), e105700 (2014).

Antonello E. Spinelli received his $\mathrm{PhD}$ in medical physics from the Institute of Cancer Research, University of London, UK, in 2004, and the medical physics expert degree from the University of Bologna in 2009. He is the small animal optical imaging research coordinator of the San Raffaele Scientific Institute, Milan, Italy. His main research areas are in vivo optical imaging, Cerenkov and radioluminescence imaging (RLI), and preclinical CT imaging. 
Giovanni Durando received his $\mathrm{PhD}$ in metrology from Politecnico di Torino, Italy, in 2003. In May 2009, he accepted a permanent position as a physicist at the Istituto Nazionale di Ricerca Metrologica in the Metrology for the Quality of Life Department. He is an active member of IEC-TC 87 (Ultrasonics) and is a delegate for the Consultative Committee for Acoustic, Ultrasound, and Vibration. His experimental activity is focused on acoustics, ultrasound, and metrology.
Federico Boschi received his $\mathrm{PhD}$ from the University of Verona in 2008 and is currently a researcher at the Optical Imaging Laboratory, University of Verona. Before his $\mathrm{PhD}$, he was involved in different research activities in astrophysics and he switched to preclinical imaging working with small animal MR and optical imaging in 2004. His main research areas are Cerenkov and RLI, in vivo optical imaging using nanoparticles, and small animal magnetic resonance imaging. 